\title{
Validez y confiabilidad del cuestionario para evaluar factores protectores de la salud mental en estudiantes universitarios
}

\section{Validity and Reliability of a Questionnaire in Exploring the Protective Factors of Mental Health in Cuban University Students}

\author{
Evelyn Fernández-Castillo ${ }^{1}$ \\ Osana Molerio-Pérez ${ }^{2}$ \\ Luis Felipe Herrera Jiménez ${ }^{3}$ \\ Ricardo $\mathrm{Grau}^{4}$ \\ Universidad Central de las Villas, Cuba
}

\begin{abstract}
Resumen. Objetivo: Analizar la validez y confiabilidad de un cuestionario para la exploración de factores protectores de la salud mental en estudiantes universitarios cubanos. Método: La muestra quedó conformada por 1377 estudiantes. Se utilizó el análisis categórico de componentes principales, el análisis de conglomerados en dos etapas y los árboles de decisión. Resultados: Se identificaron dos factores. La primera dimensión incluyó ítems que tienen un sentido positivo y expresan un desarrollo de estos factores protectores. Esta dimensión mostró un mayor coeficiente de confiabilidad y fue capaz de explicar una mayor proporción de la varianza en los clúster identificados. La segunda dimensión agrupó aquellos ítems relacionados con un pobre desarrollo de estos factores de protección que pudieran constituir en factores de riesgo. Los ítems que mejor predicen la agrupación de los tres clúster están relacionados con la resiliencia, toma de decisiones, la autoeficacia y la autoestima. Conclusión: El instrumento desarrollado mostró su exactitud y precisión en el proceso de obtención de la información necesaria para evaluar los factores de protección de la salud mental en estudiantes universitarios cubanos.
\end{abstract}

Palabras clave. Factores de protección, salud mental, estudiantes universitarios.

Abstract. Objective: To analyze the validity and reliability of a questionnaire in exploring the protective factors of mental health in Cuban university students. Method: The sample was composed of 1377 students. A categorical principal components analysis, a two-step cluster analysis and decision trees were used. Results: Results revealed two components. The first dimension included items that have a positive meaning and express a development of these protective factors. This dimension showed a higher coefficient of reliability and was able to explain a higher proportion of the variance in the clusters identified. The second dimension grouped those items that were related to poor development of these protection factors, which could constitute to risk factors. Items associated with resilience, decision making ability, self- efficacy and self- esteem were the best predictors in the grouping process. Conclusion: The instrument developed showed its accuracy and precision in the process of obtaining the necessary information to evaluate the factors of protection of mental health in Cuban university students.

Keywords. Protective factors, mental health, university students.

\footnotetext{
${ }^{1}$ Evelyn Fernández-Castillo. Universidad Central de las Villas, Cuba. Dirección Postal: Calle 2da entre A y B, \#88. Reparto Vigía, Santa Clara, Villa Clara, Cuba. Código postal: 50 200.E-mail: efernandez@uclv.edu.cu

${ }^{2}$ Osana Molerio-Pérez. Universidad Central de las Villas, Cuba. E-mail: osanam@uclvedu.cu

${ }^{3}$ Luis Felipe Herrera Jiménez. Universidad Central de las Villas, Cuba. E-mail: luisfelipehj@gmail.com

${ }^{4}$ Ricardo Grau. Universidad Central de las Villas, Cuba. E-mail: rgrau@uclv.edu.cu
}

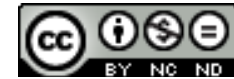

Esta obra está bajo una licencia de Creative Commons Reconocimiento-NoComercial-SinObraDerivada 4.0 Internacional. 


\section{Introducción}

La salud mental ha pasado a ocupar un lugar primordial entre las prioridades sanitarias y comunitarias en muchos países del orbe (Cuesta, Ruiz, Roca, \& Noguer, 2016; Maercker et al., 2015; OMS, 2013; Saavedra, Berenzon, \& Galván, 2016). Resulta incuestionable que no se puede hablar de salud si no se considera la salud mental. Por tanto, existe el reto profesional y social de contribuir en su fomento y en la prevención de los trastornos que pudieran afectarla desde etapas tempranas de la vida.

En los últimos años se han incrementado las investigaciones con una visión positiva de la adolescencia y la juventud (Alarcón \& Rodríguez, 2015; Giménez, Vázquez Valverde, \& Hervás Torres, 2010; Guerra \& Bradshaw, 2008; Oliva et al., 2010; Quiceno, Alpi, \& González, 2014). El modelo del desarrollo positivo (Oliva et al., 2010) refuerza la idea de que en todo adolecente o joven existen características que favorecen un desarrollo exitoso y saludable, sin abandonar el principio de que las relaciones entre el individuo y el contexto constituyen la base para el desarrollo personal. Por lo tanto, se fundamenta un claro optimismo acerca de la posibilidad de intervención; no solo para prevenir determinadas conductas indeseadas como el consumo de drogas, los problemas académicos, trastornos psicopatológicos, sino para promover estilos de vida saludables en esta etapa del desarrollo (Parra, Oliva, \& Antolín, 2009). Desde este modelo, se incorpora el concepto de recursos o activos para el desarrollo, el cual abarca recursos personales, familiares, escolares o comunitarios que proporcionan el apoyo y las experiencias necesarias para la promoción del desarrollo positivo durante la adolescencia y juventud (Oliva, Delgado, Pacheco, \& Muñoz, 2011).

Desde esta perspectiva, se han desarrollado diferentes instrumentos para evaluar estos recursos o factores de protección en adolescentes y jóvenes (González-Ramírez \& Landero 2015; Miranda-Zapata, Riquelme-Mella, Cifuentes-Cid, \& Riquelme-Bravo, 2014; Oliva, Hernando, Antolín-Suárez, \& Pascual, 2011; Salazar, Varela, Tovar, \& Cáceres, 2006; Suárez, Oliva, Pertega, \& López, 2009). Estos instrumentos se han centrado en factores de protección particulares como determinados valores, las habilidades sociales, el apoyo familiar y social. Otros han intentado explorar los factores de protección ante diferentes situaciones, como el consumo de drogas, o desde la perspectiva de los diferentes agentes educativos. Atendiendo a esto, se hace necesario contar con un instrumento que permita explorar un grupo de factores de protección para su estudio en la población universitaria cubana.

La elección de estos factores se sustentó en los supuestos del Modelo de florecimiento o desarrollo positivo (Oliva, Delgado, et al., 2011; Oliva, 2007a; Oliva et al., 2010). En este modelo, se presentan una serie de competencias específicas agrupadas en cinco bloques o áreas: emocional, social, cognitiva, moral y de desarrollo personal. De estas áreas, fueron escogidas algunas competencias para evaluarlas en el contexto universitario cubano, teniendo en cuenta la importancia de los factores culturales en su evaluación.

Las competencias escogidas en el área personal fueron: la autoestima y la autoeficacia. La primera es entendida como la valoración que el sujeto realiza de la imagen de sí mismo. No es estática, ni inamovible, no es un hecho previamente establecido. Es posible asumir que la autoestima tiene un efecto positivo en la satisfacción vital (Martínez, Buelga, \& Cava, 2007; Vera-Villarroel, Córdova-Rubio, \& Celis-Atenas, 2009). En cuanto a la autoeficacia, está entendida como creencias en las propias capacidades para organizar y ejecutar los cursos de acción requeridos que producirán determinados logros o resultados (Bandura, 1997).

En el área social, se puede hablar de las habilidades sociales; entendidas como rutinas cognitivas o conductuales que nos permiten mantener buenas relaciones con los demás. Son un tipo de herramientas personales muy útiles para un óptimo desarrollo y mantenimiento de las relaciones personales (Londoño \& Valencia, 2008; Miranda-Zapata et al., 2014). Dentro de estas se encuentran: las habilidades comunicativas o relacionales, la asertividad y las habilidades de resolución de conflictos. 
En el ámbito cognitivo, se seleccionó la capacidad para tomar decisiones, entendida como el proceso donde se identifica y analiza el problema, se buscan alternativas y se selecciona una, teniendo en cuenta los posibles resultados y sus consecuencias en el presente y el futuro (Tversky \& Kahneman, 1981). Este proceso cobra una gran importancia en la etapa en la que se enmarca la investigación, atendiendo a que se ha relacionado con la impulsividad y la implicación de adolescentes y jóvenes conductas de riesgo relacionadas con la sexualidad, el consumo de drogas o los comportamientos antisociales (BrochePérez \& Cruz-López, 2014; Oliva, 2007b).

En el área emocional, se tuvieron en cuenta el optimismo y la resiliencia. Se decidió teniendo en cuenta que las expectativas positivas con respecto a los eventos futuros (Carver \& Scheier, 2002) y la capacidad para enfrentar, sobreponerse y ser fortalecido o transformado por experiencias adversas (Grotberg, 2003) tienen una repercusión en el manejo de las emociones y en la estimulación de la motivación para alcanzar objetivos. Las investigaciones en esta etapa han explorado su relación con el éxito académico (Campa, 2010; García del Castillo \& Días, 2007; Gaxiola, González-Lugo, \& Gaxiola-Villa, 2013).

Por último, en el caso de la esfera moral se estudió, particularmente, la satisfacción del estudiante con el contexto universitario. Esto se hace a partir de sus valoraciones sobre la repercusión que ha tenido la vida universitaria para su desarrollo personal. La identificación y el estudio de estos factores de protección cobran relevancia como parte del proyecto de investigación "Metodología para la promoción de salud y el desarrollo personal desde la concepción de un Centro de Bienestar Universitario" que se desarrolla en la Universidad Central Marta Abreu de Las Villas (Fernández Castillo, Molerio, \& González-Pereira, 2016; Fernández-Castillo, Molerio, Herrera, Rodríguez-Martínez, \& Moya, 2016; Molerio, Pedraza \& Herrera, 2015). Desde el proyecto, se pretenden implementar estrategias de intervención, contextualizadas al ámbito de abordaje y que favorezcan el desarrollo de los factores en estudio para contribuir con la formación integral de los estudiantes.
Por lo tanto, como un primer paso, emerge la necesidad de desarrollar instrumentos que favorezcan el estudio de los factores protectores de la salud mental en la población referida, teniendo en cuenta la importancia que desempeñan este tipo de estudios (Roncero, 2015). Desde esta perspectiva, el presente trabajo se orientó a la construcción y el análisis de la validez y confiabilidad de un cuestionario para evaluar factores protectores de la salud mental en estudiantes universitarios cubanos.

\section{Método}

Se asumió un diseño no experimental transversal (ex-post-facto), pues se utiliza la encuesta como herramienta para la obtención de evidencia empírica en un único momento temporal. Particularmente, el presente trabajo responde a un estudio instrumental, orientado al diseño del instrumento y el estudio de su validez y confiabilidad (Montero \& León, 2007).

\section{Participantes}

La población total de estudiantes fue de 4765 estudiantes universitarios. Para la selección de la muestra se utilizó un muestreo probabilístico, considerando un error de $2.5 \%$ y un nivel de confianza de $95 \%$. La muestra quedó conformada por 1377 estudiantes universitarios. Se establecieron como criterios de inclusión: que fueran estudiantes universitarios y que accedieran voluntariamente a participar en el estudio.

El rango de edad de los estudiantes que conformaron la muestra fue de 17-34 años (635 mujeres, $M=20.3 ; D T=1.60)$. Respecto al estado civil, el 96.6\% declaró ser soltero. Formaron parte del estudio $445(32.3 \%)$ estudiantes de primer año, 314 $(22.8 \%)$ de segundo año, $316(22.9 \%)$ de tercer año y $302(21.9 \%)$ de cuarto año. En la tabla 1 se ilustra la agrupación de los estudiantes en cuanto a áreas de la ciencia. Los participantes recibieron las instrucciones por parte de los miembros del equipo de investigación, previamente capacitados. Se pidió el consentimiento informado de los participantes, con el objetivo de garantizar su voluntariedad y la confidencialidad de la información recogida. 
Tabla 1

Distribución de los estudiantes que conformaron la muestra de acuerdo a área de la ciencia

\begin{tabular}{lll}
\hline Áreas & Fr & $(\%)$ \\
\hline Ciencias Técnicas & 664 & 48.22 \\
Ciencias Naturales y Matemáticas & 161 & 11.69 \\
Ciencias Económicas & 124 & 9 \\
Ciencias Sociales y Humanísticas & 197 & 14.3 \\
Ciencias Agropecuarias & 121 & 8.78 \\
Ciencia Médicas & 110 & 8 \\
\hline
\end{tabular}

\section{Instrumentos}

Cuestionario para la evaluación de factores protectores de la salud mental en estudiantes universitarios. Se desarrolló como parte de este estudio. Permite evaluar un grupo de factores que en función de su presencia y nivel de desarrollo o alguno de los dos pueden ejercer una función protectora o de riesgo para la salud mental en estudiantes universitarios. Cuenta con 38 ítems que se responden a partir de una escala Likert del 1 al 5, donde el 1 es nunca o totalmente en desacuerdo y el 5 es siempre o totalmente de acuerdo. Los ítems: 2, 4, $8,14,15,17,25,26,31,33,34,36$ tienen calificación en inversa.

\section{Procedimiento}

Se elaboró el cuestionario para la evaluación de factores protectores de la salud mental en estudiantes universitarios, asumiendo supuestos del Modelo de florecimiento o desarrollo positivo (Oliva, Delgado, et al., 2011; Oliva, 2007a; Oliva et al., 2010). El proceso de diseño incluyó la extracción de ítems claves procedentes de instrumentos como la Escala de Autoestima (Rosenberg, 1965), la Escala de Autoeficacia Generalizada (Baessler \& Schwarzer, 1996), la Escala para la Evaluación de la Planificación y Toma de Decisiones (Darden, Ginter \& Gazda, 1996), la Escala para la Evaluación de las Habilidades Sociales (Oliva, Delgado, et al., 2011), el Test Abreviado de Orientación
Vital (Scheier, Carver, \& Bridges, 1994) y la Escala de Resiliencia (Connor \& Davidson, 2003).

Se decidió incorporar estos ítems atendiendo a que forman parte de instrumentos de evaluación cuyas propiedades psicométricas ya han sido estudiadas en la exploración de las diferentes áreas de interés para esta pesquisa. No obstante, se construyeron otros ítems en correspondencia con la definición de las categorías de interés asociadas a la esfera moral, especialmente para explorar indicadores de la satisfacción de los estudiantes con la vida universitaria.Una vez diseñado el cuestionario, se procedió a someterlo a la evaluación de especialistas. De veintidós expertos iniciales, se seleccionaron 7 por los conocimientos que tenían sobre las particularidades del contexto universitario, el diseño y la validación de instrumentos de evaluación psicológicos. En cuanto a la categoría científica, 4 fueron Doctores en Ciencias y 3 Máster. Respecto a los años de experiencia, 4 acumulaban 20 años o más; 2 iban de 11 a 19 años y solo uno tenía 5 años de experiencia. Los especialistas seleccionados analizaron el instrumento en general, respecto a la validez de contenido, la suficiencia, pertinencia y redacción de los ítems y de las opciones de respuesta. Se les solicitó que evaluaran la calidad de cada una de las proposiciones en una escala de 1 a 5 . Como tendencia, todos los ítems fueron evaluados entre 4 y 5 puntos. Se asumieron las sugerencias ofrecidas por los especialistas. 
Se realizó un estudio piloto con la aplicación del cuestionario a 30 estudiantes universitarios, con el fin de evaluar la presentación del cuestionario. Los resultados de este estudio mostraron resultados favorables; solo se modificó el ítem 38 y en este se acotó "estudiante universitario" para ubicar a los estudiantes en el rol desde el cual se le solicitaba su valoración. El tiempo de aplicación promedio no excedió los 30 minutos. Posteriormente, se procedió a su aplicación general.

\section{Resultados}

\section{Análisis}

El análisis de los datos se realizó a partir del paquete estadístico SPSS (Statistical Package for the Social Sciences), versión 15.0 para Windows. Se utilizó el análisis multivariado, específicamente, el análisis categórico de componentes principales (CATPCA, por sus siglas en inglés, Categorical Principal Component Analysis). Este procedimiento permite identificar las dimensiones que mejor representan la información que se recoge mediante las variables originales (SPSS Inc., 2007). La solución obtenida se acompaña del alfa de Cronbach para cada una de las dimensiones identificadas y para el instrumento en general.

Además, se utiliza el procedimiento de conformación de conglomerados en dos pasos (Two Step Clustering), con un criterio bayesiano de información que determina el número óptimo de conglomerados a formar (Fraley \& Raftery, 1998; Grau, 2016a; Neath \& Cavanaugh, 2012). Finalmente, se elaboró el árbol de clasificación (CHAID, por sus siglas en inglés, Chi-squared Automatic Interaction Detection), para determinar la posible relación de las variables del cuestionario y sus interacciones con los clústeres previamente formados con análisis no supervisado (Grau, 2016b).

En la tabla 2 se muestran los resultados del análisis categórico de componentes principales de las variables medidas por el cuestionario elaborado y aplicado a los 1367 estudiantes. Se identificó que la solución con dos dimensiones alcanza, de forma general, un alto índice de confiabilidad $\alpha=$.93. La primera dimensión resulta la más confiable $\alpha=.89$. La segunda dimensión aporta un alfa de Cronbach de .59, que no es despreciable.
La primera dimensión está constituida por los ítems que exploran la presencia de los factores protectores, donde se destacan los ítems que exploran la resiliencia, el optimismo, el proceso de evaluación y toma de decisiones, la autoestima y las habilidades sociales fundamentalmente. En tanto, en la dimensión dos, se agrupan los ítems que denotan poco desarrollo en estos factores de protección y que pueden ser considerados como factores de riesgo. Dentro de esta última, se destacan aquellos que exploran el pobre desarrollo de las habilidades sociales, la baja autoestima, el pesimismo, la presencia de estrés académico, el alejarse de los padres a partir de la entrada a la universidad y la percepción de que existe una oferta recreativa limitada.

El análisis de conglomerado en dos fases permitió identificar tres grupos de estudiantes, según el Criterio bayesiano de Schwarz (BIC) mostrado en la tabla 3, donde se reflejan reducciones marcadas hasta $n=3$. El primer grupo quedó integrado por estudiantes $(N=209)$ que, a partir de las respuestas ofrecidas en el cuestionario, mostraron una situación más favorable en cuanto al desarrollo y presencia de los factores protectores o alguno de los dos. El segundo conglomerado $(N=690)$ presentó resultados intermedios y el tercero agrupó a los estudiantes $(N=468)$ con un menor desarrollo o presencia de estos factores de protección.

El análisis de varianza multifactorial realizado entre las dos dimensiones identificadas anteriormente y los conglomerados previamente formados mostró, según la Traza de Hotelling, una diferencia altamente significativa esperada entre los clúster $(p<.001)$.

$\mathrm{Al}$ analizar los resultados de las pruebas de efecto intersujetos, específicamente el nivel de significación y coeficiente de determinación $\left(R^{2}\right)$, se evidencia que la primera dimensión que explora, según el análisis anterior, los factores protectores, expresa una mayor proporción de varianza explicada por los clústeres identificados $\left(p<.001, R^{2}=.732\right)$. La segunda dimensión, que explora los factores de riesgo muestra una proporción menor $\left(p<.001, R^{2}=.120\right)$.

La figura 1 presenta el árbol de clasificación construido. Permitió determinar la posible relación de los 
Tabla 2

Distribución de los items de acuerdo a las dimensiones identificadas mediante el Análisis Categórico de Componentes Principales

\begin{tabular}{|c|c|c|c|c|}
\hline \multicolumn{2}{|c|}{ Área temática que explora el ítem } & \multirow{2}{*}{$\begin{array}{l}\text { Ítems * } \\
\text { (2) Desearía sentir más aprecio por mí mismo/a }\end{array}$} & \multirow[t]{2}{*}{ D1 } & \multirow{2}{*}{$\frac{D 2}{.490}$} \\
\hline Personal & Autoestima & & & \\
\hline & & (8) Tiendo a pensar que soy un fracasado & & .471 \\
\hline & & (16) Creo que tengo muchos motivos para sentirme orgulloso/a & & \\
\hline & & (20) En general estoy satisfecho conmigo mismo/a & & \\
\hline & Autoeficacia & (1) Puedo resolver problemas difíciles si me esfuerzo lo suficiente & .506 & \\
\hline & & $\begin{array}{l}\text { (11) Si me encuentro en una situación difícil, generalmente se me ocurre qué } \\
\text { debo hacer }\end{array}$ & 619 & \\
\hline & Habilidades & (4) Me cuesta trabajo hablar cuando hay mucha gente & & .474 \\
\hline & Sociales & $\begin{array}{l}\text { (14) Siento temor empezar una conversación con alguien que me atrae } \\
\text { físicamente }\end{array}$ & & .505 \\
\hline & & (17) Me cuesta trabajo empezar una conversación con alguien que no conozco & & .499 \\
\hline & & (21) Suelo mediar en los problemas entre compañeros/as & .385 & \\
\hline & & $\begin{array}{l}\text { (23) Me gusta decirle a una persona que estoy muy satisfecho/a por algo que } \\
\text { ha hecho }\end{array}$ & .508 & \\
\hline \multirow[t]{4}{*}{ Cognitiva } & Toma de & (5) Considero todos los factores para tomar una decisión ante un problema & & \\
\hline & Decisiones & $\begin{array}{l}\text { (7) Soy capaz de diseñar un buen plan de acción cuando tengo que decidir algo } \\
\text { importante }\end{array}$ & .576 & \\
\hline & & $\begin{array}{l}\text { (9) Al tomar una decisión, cuando pienso en varias opciones, las comparo con } \\
\text { detalle }\end{array}$ & .503 & \\
\hline & & $\begin{array}{l}\text { (36) Generalmente no analizo cómo mis decisiones actuales pueden afectar mis } \\
\text { metas futuras }\end{array}$ & -.377 & \\
\hline \multirow[t]{11}{*}{ Emocional } & Optimismo & (15) No espero que cosas buenas me sucedan & & .374 \\
\hline & & (24) En tiempos de incertidumbre, tiendo a pensar que me va a ocurrir lo mejor & & \\
\hline & & (25) Rara vez espero que las cosas salgan a mi manera & -.427 & \\
\hline & & (26) Si algo malo me puede pasar, estoy segura(o) que me pasará & & .428 \\
\hline & & (29) Siempre soy optimista en cuanto al futuro & & \\
\hline & Resiliencia & (3) Bajo presión, me mantengo enfocado/a y pienso claramente & .454 & \\
\hline & & (6) Creo que puedo lograr mis objetivos, incluso si hay obstáculos & & .596 \\
\hline & & (10) No me desanimo fácilmente ante el fracaso & .478 & \\
\hline & & (12) Cuando me enfrento a los problemas intento ver su lado cómico & & \\
\hline & & (13) Puedo enfrentarme a cualquier cosa & .493 & \\
\hline & & (18) Enfrentarme a las dificultades puede hacerme más fuerte & & \\
\hline
\end{tabular}

Continúa...

Actualidades en Psicología, 31(122), 2017, 103-117 


\begin{tabular}{|c|c|c|c|c|}
\hline \multirow{5}{*}{\multicolumn{2}{|c|}{ Área temática que explora el ítem }} & Ítems * & D1 & D2 \\
\hline & & $\begin{array}{l}\text { (22) Tengo tendencia a recuperarme pronto luego de enfermedades, heridas } \\
\text { u otras dificultades }\end{array}$ & .465 & \\
\hline & & $\begin{array}{l}\text { (27) Soy capaz de manejar sentimientos desagradables/dolorosos: Ej. tristeza, } \\
\text { temor y enfado }\end{array}$ & & \\
\hline & & (28) Soy capaz de adaptarme cuando surgen cambios & .518 & \\
\hline & & $\begin{array}{l}\text { (30) Creo que soy una persona fuerte cuando me enfrento a los desafíos y } \\
\text { dificultades vitales }\end{array}$ & 690 & \\
\hline \multirow[t]{7}{*}{ Social } & \multirow{7}{*}{$\begin{array}{l}\text { Satisfacción } \\
\text { personal con } \\
\text { el contexto } \\
\text { universitario }\end{array}$} & (31) Las demandas académicas de la universidad a menudo me estresan & & \multirow[t]{2}{*}{.396} \\
\hline & & $\begin{array}{l}\text { (32) Siento que me puedo acercar a mis profesores para conversar sobre } \\
\text { cualquier problema que tenga }\end{array}$ & .373 & \\
\hline & & $\begin{array}{l}\text { (33) Desde que ingresé en la universidad me he alejado de mis padres, incluso } \\
\text { afectivamente }\end{array}$ & & .283 \\
\hline & & $\begin{array}{l}\text { (34) Considero que la oferta de actividades recreativas de la universidad es } \\
\text { muy limitada }\end{array}$ & & \multirow[t]{4}{*}{.163} \\
\hline & & $\begin{array}{l}\text { (35) Para mí la vida universitaria ha significado un proceso continuo de } \\
\text { estudio, cambio y crecimiento }\end{array}$ & .425 & \\
\hline & & $\begin{array}{l}\text { (37) Aunque paso mucho tiempo en la universidad, comparto mis logros y } \\
\text { dificultades con mis padres }\end{array}$ & .434 & \\
\hline & & $\begin{array}{l}\text { (38) Si tuviera que evaluar el grado satisfacción que experimento como } \\
\text { estudiante universitario lo ubicaría en el nivel... }\end{array}$ & .470 & \\
\hline
\end{tabular}

Nota. El número que aparece dentro del paréntesis es el que ocupa el ítem en el cuestionario, D1= Factor de protección, D2= Factor de riesgo.

factores protectores y sus interacciones con los clústeres previamente formados por el análisis no supervisado.

La tabla 4 facilita el análisis de los resultados y resume las interacciones detectadas por los 16 nodos terminales logrados con este nivel de profundidad. El ítem que mejor predice la agrupación de los estudiantes es el 30 "Creo que soy una persona fuerte cuando me enfrento a los desafíos y dificultades vitales". Esto aplica en el clúster de mejor resultado, en el de peor y en el de resultados intermedios. Le siguen los ítems: (7) "Soy capaz de diseñar un buen plan de acción cuando tengo que decidir algo importante", (28) "Soy capaz de adaptarme cuando surgen cambios", (16) "Creo que tengo motivos para sentirme orgulloso" y (11) "Si me encuentro en una situación difícil generalmente se me ocurre que debo hacer".

La matriz de confusión asociada al árbol de clasificación se muestra en la tabla 5 , donde se recoge información sobre los valores reales y las clasificaciones predichas realizadas por el árbol de clasificación. Nótese que se logra clasificar correctamente más del $70 \%$ de los casos, lo cual se considera satisfactorio y constituye un indicador de exactitud del instrumento, elemento que resulta importante tener en cuenta para evaluar su calidad.

\section{Discusión}

La propuesta realizada se basa en la importancia de contar con un instrumento, válido y confiable, que permita identificar y analizar integralmente los factores protectores de la salud mental en estudiantes universitarios cubanos. El diseño de este instrumento constituye un momento necesario para garantizar la exploración de estos factores y, consecuentemente, el desarrollo de intervenciones no solo orientadas a disminuir problemas sino también para la promoción 


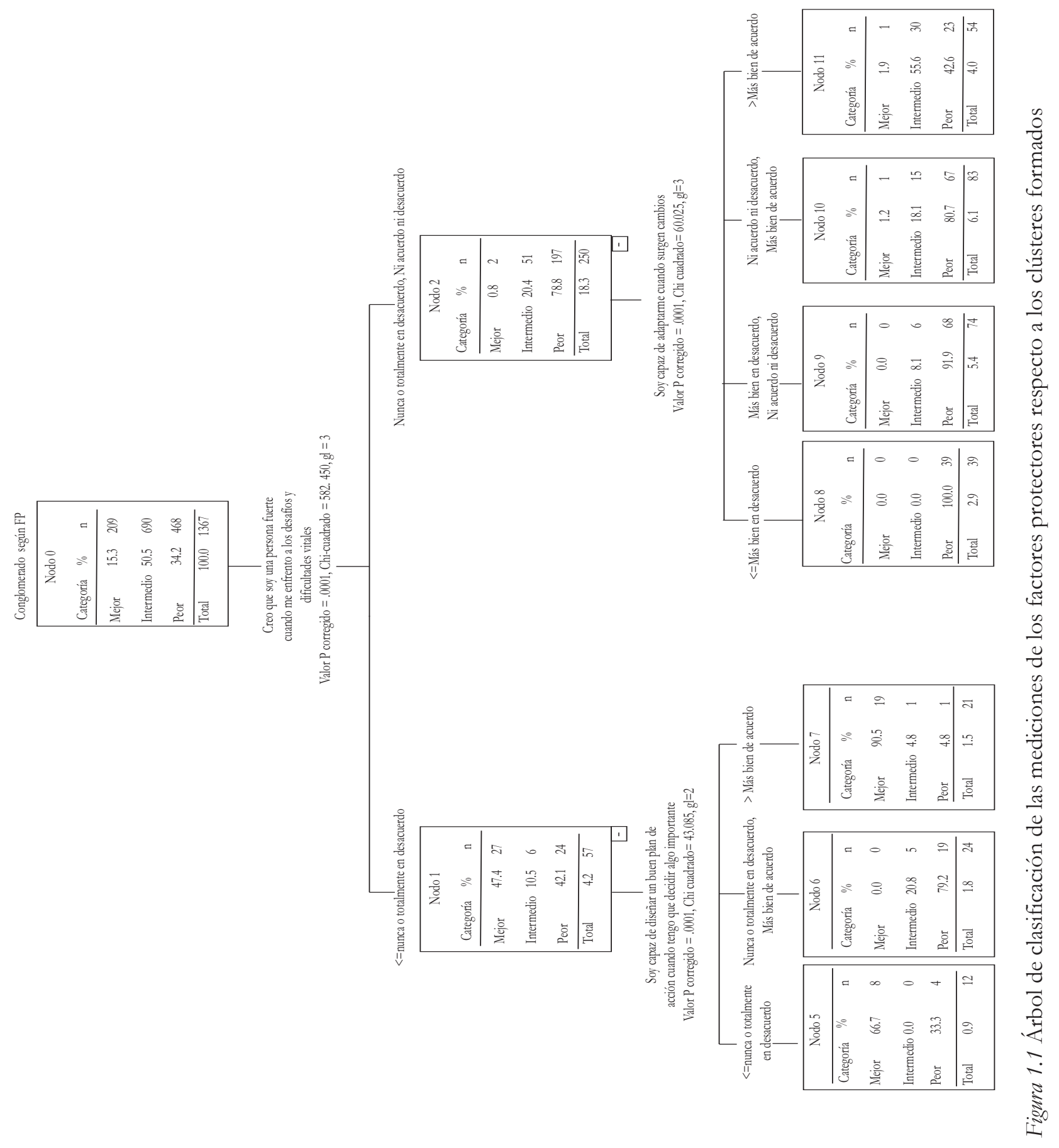




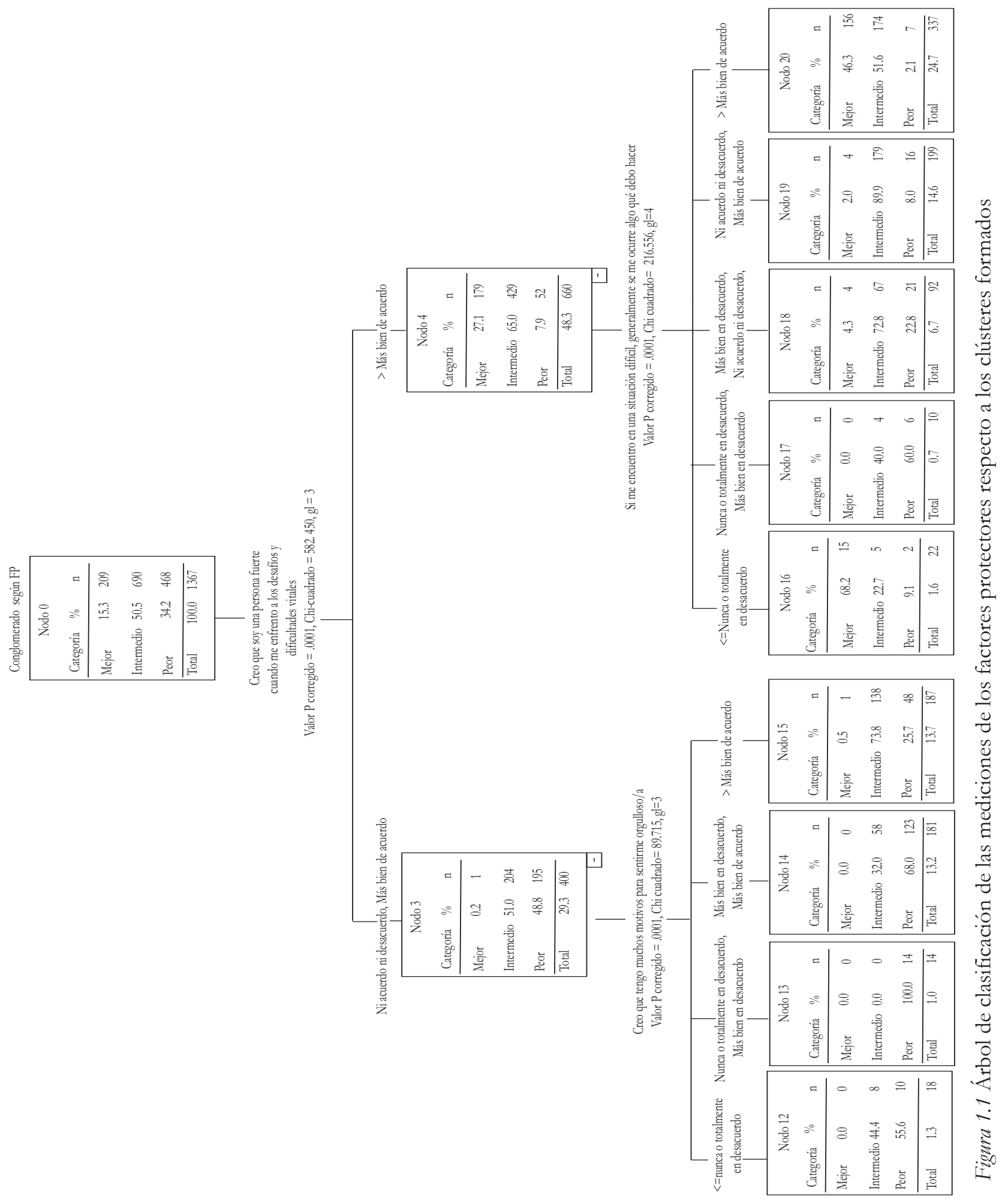


Tabla 3

Agrupación automática de los conglomerados

\begin{tabular}{cccc}
\hline Número de conglomerados & $\begin{array}{c}\text { Criterio bayesiano de } \\
\text { Schwarz (BIC) }\end{array}$ & Cambio en BIC ${ }^{\mathrm{a}}$ & Razón de cambios en BIC $^{\mathrm{b}}$ \\
\hline 1 & 140381.50 & & \\
\hline 2 & 132528.07 & -7853.43 & 1.00 \\
3 & 128738.75 & -3789.32 & .483 \\
4 & 127824.01 & -914.735 & .116 \\
5 & $\ldots$ & $\ldots$ & $\ldots$
\end{tabular}

${ }^{a}$ Los cambios proceden del número anterior de conglomerados de la tabla.

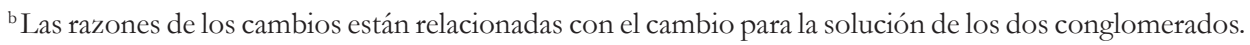

Tabla 4

Principales interacciones detectadas por el árbol de clasificación con dos niveles de profundidad.

\begin{tabular}{|c|c|c|c|}
\hline Nodo & Interacción & $\mathrm{C}$ & $F(C P)$ \\
\hline 5 & $\begin{array}{l}\text { "Creo que soy una persona fuerte cuando me enfrento a los desafíos y dificultades vitales" = Nunca } \\
\text { o totalmente en desacuerdo y "Soy capaz de diseñar un buen plan de acción cuando tengo que decidir } \\
\text { algo importante" = Nunca }\end{array}$ & Mejor & $33.3 \%$ \\
\hline 6 & $\begin{array}{l}\text { "Creo que soy una persona fuerte cuando me enfrento a los desafíos y dificultades vitales" = Nunca } \\
\text { o totalmente en desacuerdo y "Soy capaz de diseñar un buen plan de acción cuando tengo que decidir } \\
\text { algo importante" = Más bien en desacuerdo - Más bien de acuerdo }\end{array}$ & Peor & $79.2 \%$ \\
\hline 7 & $\begin{array}{l}\text { "Creo que soy una persona fuerte cuando me enfrento a los desafíos y dificultades vitales" = Nunca } \\
\text { o totalmente en desacuerdo y "Soy capaz de diseñar un buen plan de acción cuando tengo que decidir } \\
\text { algo importante" = Siempre o totalmente de acuerdo }\end{array}$ & Mejor & $4.8 \%$ \\
\hline 8 & $\begin{array}{l}\text { "Creo que soy una persona fuerte cuando me enfrento a los desafíos y dificultades vitales" = Más } \\
\text { bien en desacuerdo - Ni acuerdo ni desacuerdo y Soy capaz de adaptarme cuando surgen cambios" = } \\
\text { Nunca - Más bien en desacuerdo }\end{array}$ & Peor & $100.0 \%$ \\
\hline 9 & $\begin{array}{l}\text { "Creo que soy una persona fuerte cuando me enfrento a los desafíos y dificultades vitales" = Más bien } \\
\text { en desacuerdo - Ni acuerdo ni desacuerdo y "Soy capaz de adaptarme cuando surgen cambios" = Ni } \\
\text { acuerdo ni desacuerdo }\end{array}$ & Peor & $91.9 \%$ \\
\hline 10 & $\begin{array}{l}\text { "Creo que soy una persona fuerte cuando me enfrento a los desafíos y dificultades vitales" = Más bien } \\
\text { en desacuerdo - Ni acuerdo ni desacuerdo y "Soy capaz de adaptarme cuando surgen cambios" = Más } \\
\text { bien de acuerdo }\end{array}$ & Peor & $80.7 \%$ \\
\hline 11 & $\begin{array}{l}\text { "Creo que soy una persona fuerte cuando me enfrento a los desafíos y dificultades vitales" = Más bien } \\
\text { en desacuerdo - Ni acuerdo ni desacuerdo y "Soy capaz de adaptarme cuando surgen cambios" = } \\
\text { Siempre o totalmente de acuerdo }\end{array}$ & Intermedio & $42.6 \%$ \\
\hline 12 & $\begin{array}{l}\text { "Creo que soy una persona fuerte cuando me enfrento a los desafíos y dificultades vitales" = Más bien } \\
\text { de acuerdo y "Creo que tengo muchos motivos para sentirme orgulloso/a" = Nunca o totalmente en } \\
\text { desacuerdo }\end{array}$ & Peor & $55.6 \%$ \\
\hline
\end{tabular}



de acuerdo y "Creo que tengo muchos motivos para sentirme orgulloso/a" = Más bien en desacuerdo

14 "Creo que soy una persona fuerte cuando me enfrento a los desafíos y dificultades vitales" = Más bien de acuerdo y "Creo que tengo muchos motivos para sentirme orgulloso/a" $=\mathrm{Ni}$ acuerdo ni desacuerdo - Más bien de acuerdo

15 "Creo que soy una persona fuerte cuando me enfrento a los desafíos y dificultades vitales" = Más bien de acuerdo y "Creo que tengo muchos motivos para sentirme orgulloso/a" = Siempre o totalmente de acuerdo

16 "Creo que soy una persona fuerte cuando me enfrento a los desafíos y dificultades vitales" = Siempre o totalmente de acuerdo \& "Si me encuentro en una situación difícil, generalmente se me ocurre qué debo hacer" = Nunca o totalmente en desacuerdo

17 "Creo que soy una persona fuerte cuando me enfrento a los desafíos y dificultades vitales" = Siempre o totalmente de acuerdo y "Si me encuentro en una situación difícil, generalmente se me ocurre qué debo hacer" = Más bien en desacuerdo

18 "Creo que soy una persona fuerte cuando me enfrento a los desafíos y dificultades vitales" = Siempre o totalmente de acuerdo y "Si me encuentro en una situación difícil, generalmente se me ocurre qué debo hacer" = Ni acuerdo ni desacuerdo

19 "Creo que soy una persona fuerte cuando me enfrento a los desafíos y dificultades vitales" = Siempre o totalmente de acuerdo y "Si me encuentro en una situación difícil, generalmente se me ocurre qué debo hacer"= Más bien de acuerdo

20 "Creo que soy una persona fuerte cuando me enfrento a los desafíos y dificultades vitales" = Siempre o totalmente de acuerdo y "Si me encuentro en una situación difícil, generalmente se me ocurre qué debo hacer" = Siempre o totalmente de acuerdo

Nota. $\mathrm{C}=$ Clúster más frecuente, F; (CP)=Frecuencia en el clúster de peor resultado.

Tabla 5

Matriz de confusión asociada al árbol de clasificación

\begin{tabular}{|c|c|c|c|c|}
\hline \multirow{2}{*}{ Observado } & \multirow[b]{2}{*}{ Mejor } & \multicolumn{2}{|c|}{ Pronosticado } & \multirow[b]{2}{*}{ Porcentaje correcto } \\
\hline & & Intermedio & Peor & \\
\hline Mejor & 42 & 166 & 1 & $20.1 \%$ \\
\hline Intermedio & 6 & 588 & 96 & $85.2 \%$ \\
\hline Peor & 7 & 115 & 346 & $73.9 \%$ \\
\hline Porcentaje Global & $4.0 \%$ & $63.6 \%$ & $32.4 \%$ & $71.4 \%$ \\
\hline
\end{tabular}

Método de crecimiento: CHAID.

Variable dependiente: Conglomerados identificados. 
de conductas saludables. Esta perspectiva, se configura como una tarea prioritaria en el escenario de la educación superior y, especialmente, para el Centro de Bienestar Universitario perteneciente a la Universidad Central "Marta Abreu" de Las Villas, contexto donde se desarrolló la investigación.

El resultado del análisis factorial permitió identificar una solución de dos componentes, con adecuados índices de confiabilidad. En el primer componente se incluyeron ítems que tienen un sentido positivo y expresan un desarrollo de estos factores protectores. Mostró un mayor coeficiente de confiabilidad y fue capaz de explicar una mayor proporción de la varianza en los clúster identificados con respecto a la segunda dimensión.

El segundo componente quedó integrado por ítems que se relaciona con un pobre desarrollo de estos factores de protección, que pudieran constituir en factores de riesgo y que han sido documentados en la literatura. Podrían ser el pobre desarrollo de las habilidades sociales, la baja autoestima, el pesimismo, la presencia de estrés académico, el alejarse de los padres a partir de la entrada a la universidad y la percepción de que existe una oferta recreativa limitada (Carballo et al., 2004; Pascucci, 2015; Salazar et al., 2006).

La exactitud y precisión del instrumento también se pudo explorar a partir de la obtención de un árbol de decisión que logró más de un $70 \%$ de buena clasificación. Este análisis permitió identificar como predictores de la agrupación de los estudiantes en los diferentes clúster, los ítems relacionados con áreas temáticas relacionadas con la resiliencia, la toma de decisiones, la autoestima y la autoeficacia. Diferentes investigaciones han apuntado la relación entre estas competencias y diferentes problemáticas presentes en la población joven (García del Castillo, García del Castillo-López, Gázquez \& Marzo, 2013; GarcíaSoriano, Belloch \& Castañeiras, 2010; Gaxiola et al., 2013; Quiceno et al., 2014; Salanova, Martínez, Bresó, Llorens \& Grau, 2005). Este análisis evidenció la correspondencia entre los ítems y componentes identificados mediante el análisis factorial.
De forma general, el análisis de los datos obtenidos mostró evidencias de la calidad del instrumento en el proceso de obtención de la información necesaria para evaluar los factores de protección de la salud mental en estudiantes universitarios cubanos. No obstante, se considera que el presente trabajo constituye una primera aproximación al estudio de las propiedades psicométricas de este cuestionario. Se sugiere que futuras investigaciones profundicen en el estudio de sus evidencias de validez.

Además, es recomendable emplear la información que se obtiene a partir de este cuestionario, para desarrollar estrategias de intervención con mayores niveles de efectividad ajustadas a las necesidades reales de los jóvenes. Así, se contribuye a que el paso por la universidad se convierta en un contexto de crecimiento, desarrollo personal y preparación para la vida.

\section{Referencias}

Alarcón, R., \& Rodríguez, T. C. (2015). Relaciones entre gratitud y felicidad en estudiantes universitarios de Lima metropolitana. Psychologia: Avances de La Disciplina, 9(1), 59-69. Recuperado de: http:// revistas.usbbog.edu.co/index.php/Psychologia/ article/view/592

Baessler, J., \& Schwarzer, R. (1996). Evaluación de la autoeficacia: adaptación española de la escala de autoeficacia general. Ansiedad Y Estrés, 2(1), 1-8.

Bandura, A. (1997). Self-efficacy: The exercise of control. New York: NY: W.H. Freeman \& Company.

Broche-Pérez, Y., \& Cruz-López, D. (2014). Toma de decisiones en la adolescencia: Entre la razón y la emoción . Ciencia Cognitiva, 8(3), 70-72.

Campa, B. (2010). Critical Resilience, Schooling Processes, and the Academic Success of Mexican Americans in a Community College. Hispanic Journal of Behavioral Sciences, 32(3), 429-455. doi: 10.1177/0739986310369322

Carballo, J. L., García, O., Secades, R., Fernández, J. R., García- Cueto, E., Errasti, J. M., \& AlHalabi, S. (2004). Construcción y validación de un 
cuestionario de factores de riesgo interpersonales para el consumo de drogas en la adolescencia. Psicothema, 16(4), 674-679.

Carver, C. S., \& Scheier, M. F. (2002). Optimism. In C. R. Snyder \& S. J. Lopez (Eds.), Handbook of Positive Psychology (pp. 231-243). UK: Oxford University Press.

Connor, K. M., \& Davidson, J. R. T. (2003). Development of a new resilience scale: the ConnorDavidson Resilience Scale (CD-RISC). Depression and Anxiety, 18, 71-82.

Cuesta, J. de P., Ruiz, J. S., Roca, M., \& Noguer, I. (2016). Salud mental y salud pública en España: vigilancia epidemiológica y prevención. Psiquiatría Biológica, 23(2), 67-73. doi: 10.1016/j. psiq.2016.03.001

Darden, C. A., Ginter, E. J., \& Gazda, G. M. (1996). Life skills development scale - adolescent form: The theoretical and therapeutic relevance of life-skills. Journal of Mental Health Counseling, 18, 142-163.

Fernández Castillo, E., Molerio, O., \& GonzálezPereira, I. (2016). La educación para la salud como parte de la estrategia educativa: experiencias desde el centro de bienestar universitario. In Congreso Internacional Universidad 2016. La Habana.

Fernández-Castillo, E., Molerio, O., Herrera, L. F., Rodríguez- Martínez, Y., \& Moya, T. (2016). El bienestar universitario y su compromiso con la calidad de vida de la sociedad. En VII Convención Internacional de Psicología. Hominis 2016. La Habana.

Fraley, C., \& Raftery, A. E. (1998). How many clusters? Which clustering method? Answers via model-based cluster analysis. Computer Journal, 4, 578-588.

García del Castillo, J. A., \& Días, P. (2007). Análisis relacional entre los factores de protección, resiliencia, autorregulación y consumo de drogas. Health and Addictions, 7(2), 309-332.

García del Castillo, J. A., García del CastilloLópez, A., Gázquez, M., \& Marzo, J. C. (2013).
Emotional intelligence as an addiction prevention strategy. Addicciones, 13(2), 89-97. doi: 10.13140/ RG.2.1.5130.7047

García-Soriano, G., Belloch, A., \& Castañeiras, C. (2010). Áreas en las que basamos nuestra autoestima y su influencia sobre el bienestar emocional: Validación de la escala de contingencias de la autoestima para su uso en castellano. Revista Argentina de Clínica Psicológica, XIX(1890), 5-13.

Gaxiola, J. C., González-Lugo, S., \& Gaxiola-Villa, E. (2013). Autorregulación, Resiliencia y Metas Educativas : Variables Protectoras del Rendimiento Académico de Bachilleres. Revista Colombiana de Psicología, 22(2), 241-252.

Giménez, M., Vázquez Valverde, C., \& Hervás Torres, G. (2010). El análisis de las fortalezas psicológicas en la adolescencia: Más allá de los modelos de vulnerabilidad. Psychology, Society \& Education, 2(2), 97-116. Recuperado de: http://dialnet.unirioja.es/ servlet $/$ articulo?codigo $=3445211 \&$ info $=$ resumen \&idioma $=\mathrm{ENG}$

González- Ramírez, M. T., \& Landero, R. (2015). Propiedades Psicométricas de la Escala de Apoyo Social Familiar y de Amigos (AFA-R) en una muestra de estudiantes. Acta de Investigación Psicológica, 4(2), 1469-1480.

Grau, R. (2016a). Bayesian Information Criterion in two step clustering. Santa Clara.

Grau, R. (2016b). Matrices de confusión y curvas ROC. Santa Clara.

Grotberg, E. (2003). Resiliencia, descubriendo las propias fortalezas. Paidós.

Guerra, N. G., \& Bradshaw, C. P. (2008). Linking the prevention of problem behaviors and positive youth development: Core competencies for positive youth development and risk prevention. In N. G. Guerra \& C. P. Bradshaw (Eds.), Core competencies to prevent prob-lem behaviors and promote positive youth development. New (pp. 1-17). http:// doi.org/10.1002/cd 
Londoño, C., \& Valencia, C. (2008). Asertividad, resistencia a la presión de grupo y consumo de alcohol en universitarios. Acta Colombiana de Psicología, 11(1), 155-162.

Maercker, A., Chi, X., Gao, Z., Kochetkov, Y., Lu, S., Sang, Z., ... Margraf, J. (2015). Personal value orientations as mediated predictors of mental health: A three-culture study of Chinese , Russian, and German university students. International Journal of Clinical and Health Psychology, 15(1), 8-17. doi: 10.1016/j.ijchp.2014.06.001

Martínez, M., Buelga, S., \& Cava, M. J. (2007). La satisfacción con la vida en la adolescencia y su relación con la autoestima y el ajuste escolar. Anuario de Psicología, 38(2), 293-303.

Miranda-Zapata, E., Riquelme-Mella, E., CifuentesCid, H., \& Riquelme-Bravo, P. (2014). Análisis factorial confirmatorio de la Escala de habilidades sociales en universitarios chilenos. Revista Latinoamericana de Psicología, 46(2), 7382. doi: 10.1016/S0120-0534(14)70010-X

Molerio, O., Pedraza, L., \& Herrera, L. F. (2015). Universidades por la salud: experiencias desde la implementación de un centro de bienestar universitario. Congreso Universidad, IV(1), 45-57.

Montero, I., \& León, O. G. (2007). Guía para nombrar los estudios de investigación en Psicología. International Journal of Clinical and Health Psychology, 7(3), 847-862.

Neath, A. A., \& Cavanaugh, J. E. (2012). The Bayesian information criterion: background, derivation, and applications. WIREs Comp Stat, 4, 199-203.

Oliva, A. (2007a). Adolescencia en positivo. Apuntes de Psicología, 25(3), 235-237.

Oliva, A. (2007b). Desarrollo cerebral y asunción de riesgos durante la adolescencia. Apuntes de Psicología, 25(3), 239-254.

Oliva, A., Delgado, I., Pacheco, R., \& Muñoz, L. (2011). Desarrollo positivo adolescente y los activos que lo promueven. Un estudio en centros docentes andaluces. Junta de Andalucía. Consejería de Salud.
Oliva, A., Hernando, A., Antolín-Suárez, L., \& Pascual, D. (2011). Activos para el desarrollo positivo y la salud mental en la adolescencia. Junta de Andalucía. Consejería de Salud.

Oliva, A., Ríos, M., Antolín-Suárez, L., Parra, A., Hernando, A., \& Pertegal, M. A. (2010). Más allá del déficit: construyendo un modelo de desarrollo positivo adolescente. Infancia Y Aprendiraje, 32(2), 223-234. doi: 10.1174/021037010791114562

OMS. (2013). Plan de Acción sobre Salud Mental 20132020. Ginebra: Autor.

Parra, Á., Oliva, A., \& Antolín, L. (2009). Los programas extraescolares como recurso para fomentar el desarrollo positivo adolescente. Papeles Del Psicólogo, 30(3), 182-183.

Pascucci, M. (2015). Los jóvenes universitarios y el ocio. European Scientific Journal, 11(10). Recuperado de: http://eujournal.org/index.php/esj/article/ view/5551

Quiceno, J. M., Alpi, S. V., \& González, C. (2014). Influencia de los factores salutogénicos y patogénicos sobre la calidad de vida de adolecentes colombianos. Revista Argentina de Clínica Psicológica, XXIII, 81-93.

Roncero, C. (2015). La validación de instrumentos psicométricos: un asunto capital en la salud mental. Salud Mental, 38(4), 235-236. doi: 10.1177/1087054714529819

Rosenberg, M. (1965). Society and Adolescent self-image. Princeton: University Press.

Saavedra, N., Berenzon, S., \& Galván, J. (2016). Atención Primaria Salud mental y atención primaria en México. Posibilidades y retos. Atención Primaria, 48(4), 258-264. doi: 10.1016/j.aprim.2015.05.005

Salanova, M., Martínez, I. M., Bresó, E., Llorens, S., \& Grau, R. (2005). Bienestar psicológico en estudiantes universitarios: facilitadores y obstaculizadores del desempeño académico. Anales de Psicología, 21(1), 170-180. Recuperado de: http:// revistas.um.es/analesps/article/view/27261 
Salazar, C., Varela, M. T., Tovar, J. R., \& Cáceres, D. E. (2006). Construcción y validación de un cuestionario de factores de riesgo y de protección para el cosnumo de drogas en jóvenes universiatrios. Acta Colombiana de Psicología, 9(2), 19-30.

Scheier, M. F., Carver, C. S., \& Bridges, M. W. (1994). Distinguising optimism from neuroticism (and trait anxiety, self mastery and self esteem): A reevaluation of the Life Orientation Test. Journal of Personality and Social Psychology, 67, 1063-1078.

SPSS Inc. (2007). Categorical Principal Components Analysis (CATPCA). In SPSS Categories 17.0 (p. 35).
Suárez, L. A., Oliva, A., Pertega, M. A., \& López, A. M. (2009). Desarrollo y validación de una escala de valores para el desarrollo positivo adolescente. Psicothema, 23(1), 153-159.

Tversky, A., \& Kahneman, D. (1981). The Framing of Decisions and the Psychology of Choice. Science, 211, 453-458.

Vera-Villarroel, P., Córdova-Rubio, N., \& CelisAtenas, K. (2009). Optimismo Versus Autoestima: Implicancia para la psicología clínica y psicoterapia. Revista Argentina de Clínica Psicológica, XVIII, 21-30.

Recibido: 19 de mayo de 2016 Aceptado: 19 de octubre de 2016 
